\title{
Consórcio de Micro-organismos e seus Efeitos sobre a Produtividade de Vagens do Amendoim
}

\author{
Submetido - 27 jul. 2020 \\ Aprovado - 08 ago. 2020 \\ Publicado - 14 set. 2020
}

http://dx.doi.org/10.17648/sas.v1i2.34

Denizart Bolonhezi (D)

Pesquisador Científica do Instituto Agronômico de Campinas - IAC, Centro Avançado de Pesquisa em Cana, Ribeirão Preto, SP, e-mail: denizart@iac.sp.gov.br.

\section{Larissa Moraes da Silva Ambrósio (D)}

Engenheira Agrônoma - Bolsista de Aperfeiçoamento da FUNDAG, IAC, Centro de Cana, Ribeirão Preto, SP e-mail: larisaambrosio01@gmail.com.

Pós-graduando, Universidade Estadual Paulista - UNESP/FCAV, Jaboticabal - SP, e-mail: olavobetiol96@gmail.com.

André Luiz Biagini Totino (iD

Graduando em Agronomia, Bolsista PIBIC/IAC - Instituto Moura Lacerda, Ribeirão Preto -SP, e-mail: andrebiaginitotino@outlook.com. le.palviqueres@gmail.com.

Engenheiro Agrônomo, Mestrando em Agronomia - Departamento de Solos, FCAV/Unesp, Jaboticabal, SP; e-mail: elciorpleal@gmail.com.

Renato Melo Martins F. Silva (iD)

Engenheiro Agrônomo, Bolsista de Aperfeiçoamento da FUNDAG, Ribeirão Preto, SP, -mail: dbolonhezi@gmail.com.

\section{RESUMO}

O uso de insumos à base de micro-organismos é uma forte tendência na agricultura contemporânea, com propósitos fitossanitários, nutricionais e de aumento da tolerância aos estresses abióticos, embora ainda incipiente na cultura do amendoim. Com objetivo de avaliar os efeitos agronômicos do consórcio de micro-organismos foram instalados três ensaios, sendo um em casa-de-vegetação no ano 2018 e dois em campo na safra 2018/2019 (reforma de canavial) e 2019/2020 (em sucessão ao milho) em condição de Latossolo Vermelho eutrófico, na área experimental do Centro de Cana do IAC, Ribeirão Preto/SP. Em todos os ensaios utilizou-se delineamento experimental em blocos casualizados, sendo 03 tratamentos (Testemunha, Bacillus pumilus e SoloPrêmio ${ }^{\circledR}$ ). No ensaio em condição de casade-vegetação (cv IAC-OL3); 05 tratamentos (Testemunha, Bacillus subtilis, Bacillus pumilus, B. subtilis + B. pumilus, SoloPrêmio ${ }^{\circledR}$ ) na safra 2018/19 (cv IAC-503) e 04 tratamentos (Testemunha, SoloPrêmio ${ }^{\circledR}$ e dois consórcios experimentais C1 e C2) na safra 2019/2020 (cV IAC-OL3). $O$ consórcio 1 (Azospirilum sp., Bacillus subtilis, Bacillus thuringiensis, Bradyrhizobium japonicum) e consórcio 2 (Bacillus pumillus, Bacillus amilolyquefaciens e Trichoderma sp.) não são comerciais. Em condições controladas foi possível identificar aumento de $46 \%$ no número de nódulos, na safra 2018/19 (ocorrência de veranicos) ganhos de $16 \%$ na produtividade de vagens e na safra $2019 / 20$ aumentos expressivos de $17 \%$ na biomassa seca total aos 90 dias, quando foram aplicados micro-organismos isoladamente e em consórcio. Pode-se concluir que o uso de consórcio de micro-organismos é uma estratégia importante sobretudo em condições adversas de clima e solo.

Palavras-chave: Arachis hypogaea L.; Insumos biológicos; Grãos. 


\title{
Consortium of Microorganism and Their Effects on Peanut Pod Yield
}

\begin{abstract}
The objective of this study was to evaluate yield and pod maturity condition in peanut lines of the runner type, so as to indicate cultivars with reduced cycle. Maturity assesment was evaluated in two sampling dates before harvesting. The experiment was carried out in Ribeirão Preto, SP, Brasil, in randomized blocks design, testing tem breeding lines and two control cultivars. Pod maturity was evaluated at 119 and 125 days after planting. In each sampling date the plants contained in $0,5 \mathrm{~m}$ of row were collected and pod maturity was determined based in a maturity table considering the color of the pod mesocarp, in a scale varying from white to black. Harvesting to evaluate yield was done at 125 days after planting, by digging one of the two lines of each plot. Along with pod yield, the following variables were obtained: percentage of the number of mature pods in each sampling date and the percentage of increase in mature pods from the 119 to 125 sampling dates. The results indicated that, when harvesting at 125 days, the line 13.24 as the most productive (7,625 $\left.\mathrm{kg} \mathrm{ha}^{-1}\right)$. At 119 days after planting, the line 13.34 showed the highest percentage of mature pods $(56.1 \%)$. The lines 13.15 and 13.24 showed the largest increases in number of mature pods from the 119th to the 125th day after planting. The results indicate that there are promising lines for selection as new cultivars combining productivity and earliness, to be confirmed in other environments.
\end{abstract}

Keywords: Arachis hypogaea L.; Biological products; Kernels.

\section{Consórcio de los Microrganismos y Sus Efectos sobre la Productividad de la Vaina del Maní}

\section{RESUMEN}

El uso del consórcio de microorganismos es una tendencia fuerte en la agricultura contemporánea, con una fitosanitaria, nutricional y una mayor tolerancia al estrés abiótico, aunque todavía es incipiente en el cultivo de maní. Con el objetivo de evaluar los efectos agronómicos del consorcio de microorganismos, se instalaron tres ensayos, uno en un invernadero en 2018 y dos en el campo en la cosecha 2018/19 (reforma de la caña de azúcar) y 2019/20 (en sucesión al maíz)) en una condición eutrófica de Red Latosol, en el área experimental del Centro IAC Cana, Ribeirão Preto / SP. En todas las pruebas, se utilizó un diseño de bloques al azar, con 03 tratamientos (Testigo, Bacillus pumilus y SoloPrêmio ${ }^{\circledR}$ ) en la prueba de invernadero (cv IAC-OL3); 05 tratamientos (Testigo, Bacillus subtilis, Bacillus pumilus, B. subtilis + B. pumilus, SoloPrêmio ${ }^{\circledR}$ ) en la cosecha 2018/19 (cv IAC-503) y 04 tratamientos (Testigo, SoloPrêmio ${ }^{\circledR}$ y dos consórcios experimentales) en el 2019 / 20 (cv IACOL3). Bajo condiciones controladas, fue posible identificar un aumento del $46 \%$ en el número de nódulos, en la cosecha 2018/19 (lluvia abajo del normal) ganancias del 16\% en la productividad de las vainas y en la cosecha 2019/20 (Iluvia arriba del normal) incrementos del $17 \%$ en la biomasa seca total a 90 días, cuando los microorganismos se aplicaron por separado y en consorcio. Se puede concluir que el uso del consorcio del microorganismos es una estrategia importante, especialmente en condiciones adversas de clima y suelo.

Palabras claves: Arachis hypogaea L.; Productos biológicos; Granos.

\section{Introdução}

A cultura do amendoim está concentrada no Estado de São Paulo, o qual apresentou na última safra 2019/2020 148 mil ha em cultivo, produção de 524 mil toneladas e produtividade média de $3530 \mathrm{~kg} \mathrm{ha}^{-1}$ de vagens (CONAB, 2020). Essa produtividade está abaixo do custo estimado, 
que situa-se ao redor de $4125 \mathrm{~kg} \mathrm{ha}^{-1}$, demandando dos produtores a adoção de práticas e insumos agrícolas que permitam a redução dos custos e aumento da produtividade, porém em sintonia com as exigências ambientais e de segurança alimentar.

Dentre os insumos é crescente o uso de biofertilizantes. Para a agricultura mundial em geral, os biofertilizantes já representam um mercado anual superior a US $\$ 7$ bilhões, mesmo representando somente 3,4\% dos pesticidas (PARNELL et al., 2016). Existem muitas definições para biofertilizantes, mas de acordo com Vessey (2003), é considerada todo insumo que contém micro-organismos vivos, os quais quando aplicados na superfície das plantas, sementes e solos, colonizam a rizosfera ou interior dos tecidos vegetais, promovendo o crescimento devido ao aumento do suprimento ou disponibilidade de nutrientes essenciais.

Dependendo do efeito proporcionado ou mecanismo de ação, recebem outras denominações, tais como: se produz substâncias supressivas de patógenos e pragas (biopesticidas), se induz promotores de crescimento (bioestimulantes), se estimula a fixação biológica de $\mathrm{N}$ (inoculantes) ou aumenta a disponibilidade de fósforo no solo, dentre outras. Contudo, de maneira geral, esses insumos são considerados de forma genérica como fertilizantes foliares.

É importante esclarecer que a espécie Arachis hypogaea (L.) é nativa da América do Sul, fato que denota uma relação mais estreita com a biota existente nos solos. Por essa razão, os inoculantes com bactérias fixadoras de nitrogênio, testados no passado, mostravam-se pouco eficientes, pois há competição com estirpes nativas existentes nos solos.

Por outro lado, a associação com fungos micorrízicos arbusculares favorece a absorção de fósforo e permite altas produtividades mesmo em solos com baixos teores de $\mathrm{P}$, auxiliando também na proteção contra infecções por Sclerotium rolfsi (DOLEY et al., 2015; SWANDI et al., 2020). Além disso, o uso de micro-organismos antagonistas pode auxiliar na 
redução dos níveis de aflatoxinas causadas por Aspergillus flavus e Aspergillus parasiticus (REN et al., 2020), informação importante para o principal problema na cultura do amendoim, sobretudo quando o foco são as exportações.

Considerando então, que os benefícios dos biofertilizantes podem acontecer simultaneamente e em diferentes aspectos, o presente trabalho teve como objetivo; estudar os efeitos do uso de consórcio de microorganismos sobre algumas características agronômicas para os cv. IAC 503 e IAC OL-3, tanto em casa-de-vegetação quanto em campo.

\section{Material e métodos}

A pesquisa consistiu de um ensaio conduzido em condições controladas e dois em condições de campo, com solo classificado de Latossolo Vermelho eutrófico, textura argilosa, localizados em área comercial (reforma de canavial) e na área experimental da APTA/IAC em Ribeirão Preto/SP, entre 2018 e 2020.

$O$ ensaio em casa-de-vegetação foi instalado conforme delineamento em blocos ao acaso, com três tratamentos e duas repetições em cada um dos 5 blocos, totalizando 30 vasos ( $30 \mathrm{~L}$ de volume cada), os quais foram preenchidos com substrato da marca Agrinobre ${ }^{\circledR}$. Os três tratamentos foram: T1- Testemunha; T2 - Bacillus pumillus e T3 SoloPrêmio ${ }^{\circledR}$ (Azospirillum sp., Bacillus subtilis, Bacillus pumillus, Bacillus amyloliquefaciens, Bradyrhizobium japonicum e Bradyrhizobium elkani) desenvolvido pela empresa Solovita ${ }^{\circledR}$.

Os tratamentos T2 e T3 foram inoculados nas sementes do cv. IAC-OL3, imediatamente antes da semeadura nos vasos (09/03/2018) e reaplicados via pulverização após 30 dias (09/04/2018). Em cada vaso foi aplicado o volume de calda de $65 \mathrm{~mL}$ da calda preparada conforme recomendação comercial do fabricante. Na colheita realizada aos 90 DAS foram avaliadas: número de nódulos, vagens e "pegs" por planta, biomassa seca da parte aérea e raiz (submetidas a secagem em estufa de circulação de ar forçada $\pm 70^{\circ} \mathrm{C}$ ). 
$\mathrm{Na}$ safra 2018/19 foi instalado ensaio de campo comercial em reforma de canavial com 9 cortes, no município de Ribeirão Preto/SP, em solo classificado como Latossolo Vermelho eutroférrico, textura argilosa. Após preparo do solo, a cultivar de amendoim IAC-503 foi semeado no dia 30/11/2018. A adubação utilizada consistiu de 12,90 e $30 \mathrm{~kg} \mathrm{ha}^{-1}$ de $\mathrm{N}$, $\mathrm{P}_{2} \mathrm{O}_{5}$ e $\mathrm{K}_{2} \mathrm{O}$, por meio do fornecimento de $300 \mathrm{~kg} \mathrm{ha}^{-1}$ da formulação 4-3010. Os resultados da análise química da fertilidade do solo encontram-se na Tabela 1.

Tabela 1. Análise química de fertilidade do solo, após cana, referente a amostragem antes da semeadura do amendoim. Ribeirão Preto, SP, 2018.

\begin{tabular}{cccccccccc}
\hline Prof. & M.O. & $\mathrm{pH}$ & $\mathrm{P}$ & $\mathrm{K}$ & $\mathrm{Ca}$ & $\mathrm{Mg}$ & $\mathrm{SB}$ & $\mathrm{CTC}$ & $\mathrm{V} \%$ \\
\cline { 2 - 9 }$(\mathrm{cm})$ & $\mathrm{g} \mathrm{dm}^{-3}$ & ---- & $\mathrm{mg} \mathrm{dm}^{-3}$ & & \multicolumn{3}{c}{$\mathrm{mmol}_{\mathrm{c}} \mathrm{dm}^{-3}$} & & $\%$ \\
\hline $0-20$ & 20,2 & 4,3 & 23 & 1,4 & 17,5 & 7,5 & 26,4 & 74,9 & 35,3 \\
\hline
\end{tabular}

Os tratamentos foram dispostos de acordo com delineamento em blocos ao acaso e 10 repetições. Os tratamentos testados foram; T1Controle, T2- Bacillus subtillis, T3- Bacillus pumillus, T4- Bacillus subtillis + Bacillus pumillus e T5- SoloPrêmio ${ }^{\circledR}$. Foram realizadas duas aplicações, a primeira aos 35 dias e a segunda 15 dias após, utilizando pulverizador costal elétrico. Dessa vez, utilizou-se para preparo da calda, água proveniente de reservatório natural utilizada em irrigação. Os controles fitossanitários seguiram protocolo estabelecido pelo produtor parceiro. A colheita foi realizada com arrancador/invertedor e as plantas trilhadas com trilhadora de parcelas experimentais. Após secagem e limpeza, foram determinadas a biomassa seca total, produtividade de vagens, produtividade de grãos, rendimento de grãos e massa de 100 sementes.

Na safra 2019/20, ensaio de campo foi novamente instalado, porém em sucessão à cultura da soja, no município de Ribeirão Preto/SP, em solo classificado como Latossolo Vermelho eutroférrico, textura argilosa, localizado no Centro de Cana do IAC. Após preparo do solo, a cultivar de amendoim IAC-OL3 foi semeada no dia 25/11/2019, com semeadora pneumática, marca Jumil ${ }^{\circledR}$, modelo Exacta ${ }^{\circledR}$. As parcelas experimentais 
consistiram de 8 linhas de amendoim (espaçamento de 0,90 m) com 6 metros de comprimento. A área não recebeu calcário e a adubação foi de 10,75 e $25 \mathrm{~kg} \mathrm{ha}^{-1}$ de $\mathrm{N}, \mathrm{P}_{2} \mathrm{O}_{5}$ e $\mathrm{K}_{2} \mathrm{O}$, através do fornecimento de $250 \mathrm{~kg}$ ha-1 da formulação 4-30-10. Os resultados da análise da fertilidade do solo encontram-se na Tabela 2.

Tabela 2. Análise química de fertilidade do solo, após soja, referente a amostragem antes da semeadura do amendoim. Ribeirão Preto, SP, 2019.

\begin{tabular}{|c|c|c|c|c|c|c|c|c|c|}
\hline Prof. & M.O. & $\mathrm{pH}$ & P & K & $\mathrm{Ca}$ & $\mathrm{Mg}$ & SB & CTC & V\% \\
\hline$(\mathrm{cm})$ & $\mathrm{g} \mathrm{dm}^{-3}$ & ---- & $\mathrm{mg} \mathrm{dm}^{-3}$ & \multicolumn{5}{|c|}{$\mathrm{mmol}_{\mathrm{c}} \mathrm{dm}^{-3}$} & $\%$ \\
\hline $0-20$ & 3,1 & 5,5 & 50 & 8,2 & 52 & 18 & 78 & 111 & 70,3 \\
\hline
\end{tabular}

Utilizou-se delineamento experimental blocos ao acaso, com 8 repetições, sendo os tratamentos; T1- Controle, T2- SoloPrêmio ${ }^{\circledR}$, T3Consórcio-1 (composto por Azospirilum sp., Bacillus subtilis, Bacillus thuringiensis, Bradyrhizobium japonicum) e T4-Consórcio 2 (Bacillus pumillus, Bacillus amilolyquefaciens e Trichoderma sp.). As aplicações foram realizadas nos dias 20/12/2019 e 10/01/2020, utilizando pulverizador costal elétrico. A água utilizada foi proveniente de reservatório natural sem tratamento com cloro. A colheita (arranquio e inversão) foi realizada no dia $18 / 03 / 2020$ e as amostras trilhadas com trilhadora de parcelas experimentais após 3 dias de processo de cura em campo. Foram determinadas além das características agronômicas avaliadas em 2018/19, a biomassa seca total aos 90 dias após a emergência.

Os dados dos três experimentos foram submetidos à análise de variância (ANOVA) e as médias foram comparadas segundo teste de Tukey a $5 \%$ de probabilidade, utilizando o programa AgroEstat ${ }^{\circledR}$.

\section{Resultados e discussão}

Com relação ao ensaio conduzido em condições controladas (Figura 1), que as diferenças entre os tratamentos não apresentaram diferenças estatísticas, para todas as características avaliadas. Nota-se que o coeficiente de variação foi alto, fato que dificultou a identificação de 
diferenças estatísticas entre os tratamentos. Deve-se esclarecer que esse foi um trabalho preliminar e que água utilizada para irrigação recebeu tratamento com cloro, interferindo consequentemente na colonização dos micro-organismos testados. Mesmo assim, verificou-se aumento no número de nódulos $(45 \%)$ no tratamento SoloPrêmio ${ }^{\circledR}$, pois na composição estão presentes bactérias fixadoras de nitrogênio.
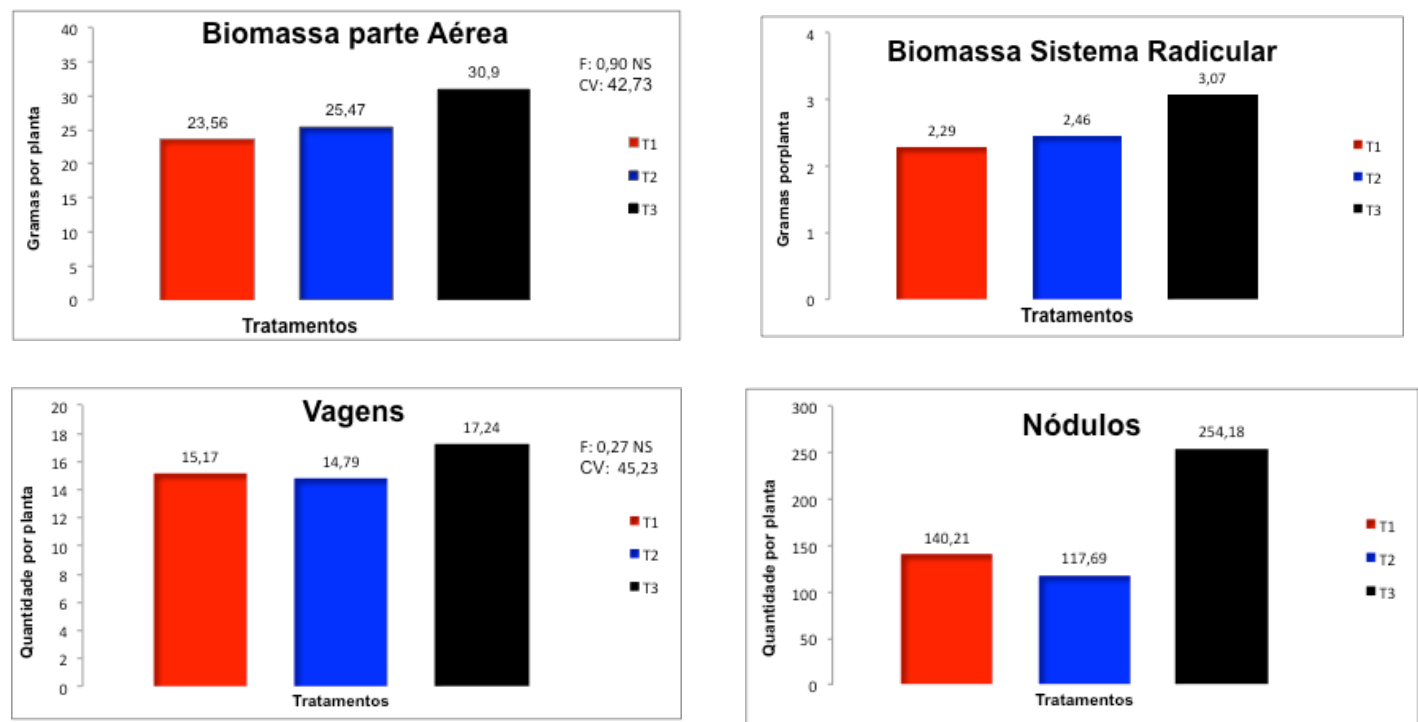

Figura 1. Resultados de biomassa seca da parte aérea e raiz, número de vagens e nódulos por planta de amendoim IAC-OL3, submetido a aplicação de microorganismos na semente e parte aérea. T1- controle; T2- Bacillus pumillus e T3- SoloPrêmio ${ }^{\circledR}$. Ensaio conduzido em casa-de-vegetação, Ribeirão Preto/SP, 2018.

$\mathrm{Na}$ Figura 2, pode-se observar os resultados de produtividade de vagens da cultivar IAC-503, referente ao ensaio de campo conduzido em condição de reforma de canavial comercial. Nota-se que houve diferença estatística ( $5 \%$ de probabilidade) para a produtividade amendoim em casca, quando se compara os tratamentos com duas aplicações isoladas dos micro-organismos Bacillus subtillis e Bacillus pumillus, bem como do consórcio SoloPrêmio ${ }^{\circledR}$, em comparação com a testemunha. O potencial produtivo foi baixo em virtude da semeadura tardia e devido a ocorrência de veranicos.

Todavia, no ensaio conduzido na safra 2019/2020 (Figura 3) constatou-se diferença estatística somente para biomassa seca total. Provavelmente, em virtude da melhor distribuição das chuvas nessa safra, 
os benefícios proporcionados pelos micro-organismos sobre os sistema radicular não tenham contribuído expressivamente.

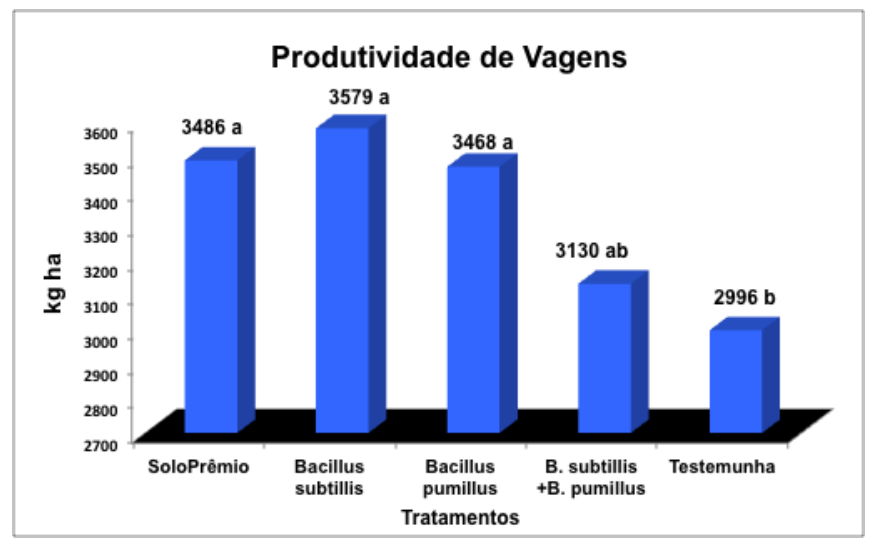

Figura 2. Produtividade de vagens do cultivar IAC-503, submetido a duas aplicações de micro-organismos isolados ou em consórcio. Valores do $C V=6,0$ e teste $F=6,36^{*}$, letras diferentes separam médias com diferença estatística (Tukey 5\%). Ribeirão Preto, SP, 2019.

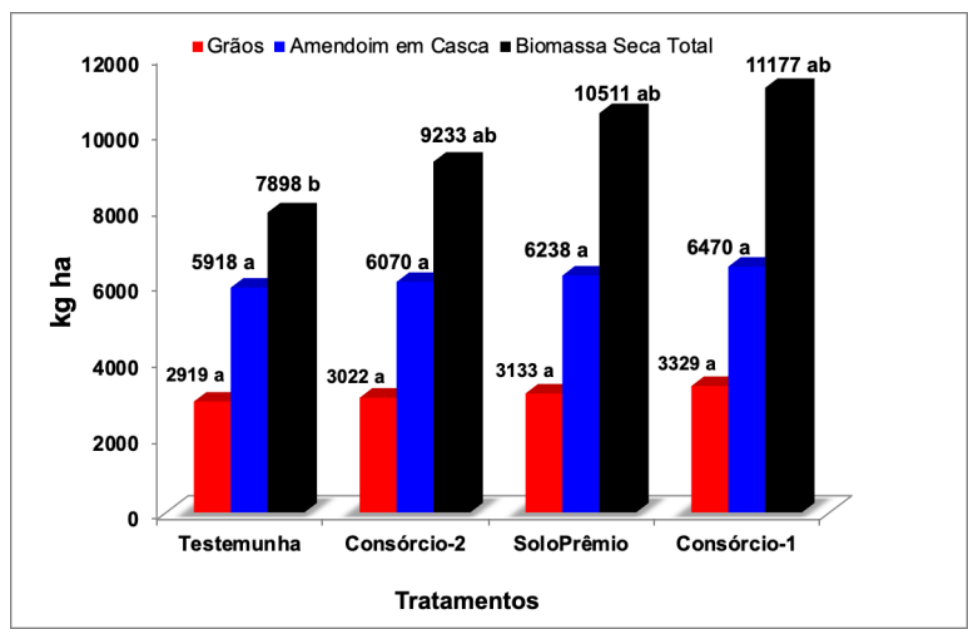

Figura 3. Produtividade de grãos (kg/ha), de amendoim em casca (kg/ha) e biomassa seca total $(\mathrm{kg} / \mathrm{ha}$ ) aos 90 dias do cultivar IAC-OL3, submetido a duas aplicações de consórcios de micro-organismos. Valores do teste $\mathrm{F}$ e CV\% foram; 1,6 ns e 12\%, 1,7 ns e 6,2\%, 2,2* e 20,4\%, respetivamente para as características grãos, amendoim em casca e biomassa seca, respectivamente. Letras diferentes separam médias com diferença estatística (Tukey 5\%). Ribeirão Preto, SP, 2019.

O Bacillus subtilis, testado isoladamente e presente no produto SoloPrêmio ${ }^{\circledR}$, é uma rizobactéria promotora de crescimento de plantas que exerce influência sobre a atividade de Rhizobium spp., aumentando a nodulação, estimulando o crescimento do sistema radicular podendo aumentar a produtividade de vagens entre 13 e 48\% (PODILE e KISHORE, 
2019). Pesquisa realizada na Índia (DEY et al., 2004), concluíram que a aplicação de nove estirpes de Pseudomonas fluorescens, aumentaram produtividade de vagens entre 18 e $28 \%$, dentre outros benefícios.

Convém salientar que o efeito negativo da aplicação combinada de Bacillus subtillis + Bacillus pumillus, denota a importância do conhecimento sobre os efeitos sinérgicos e antagonistas que ocorrem entre os microorganismos, tanto no solo quanto na rizosfera e geocarposfera (ao redor das vagens). Nas condições da Argentina, pesquisas conduzidas por Illa et al. (2020) com cultivar Granolêico, concluíram que a aplicação combinada de Trichoderma harzianum + Bacillus subtilis, aumentaram em $37 \%$ a emergência, em $46 \%$ o rendimento, em $34 \%$ o tamanho dos grãos e reduziram em $14 \%$ a incidência de Sclerotinia minor e Thecaphora frezzi (carvão do amendoim).

\section{Conclusões}

Em condições controladas, o uso de consórcio de microorganismos via semente e pulverizados na parte aérea, proporcionou aumentos de $46 \%$ na nodulação do amendoim. Em duas safras com ensaios em campo, verificou-se aumento de 16\% (496 kg ha-1) na produtividade de amendoim em casca com duas aplicações foliares de SoloPrêmio ${ }^{\circledR}$, quando ocorreu períodos de deficiência hídrica. Em ano com boa distribuição de chuva e maior fertilidade do solo, foi observado aumento de $17 \%$ na biomassa seca total aos 90 dias, sem diferenças estatísticas para produtividade (ganhos de $548 \mathrm{~kg} \mathrm{ha}^{-1}$ ).

\section{Agradecimentos}

Os autores agradecem ao CNPq pelas bolsas de produtividade DT-2 e PIBIC, bem como ao apoio da empresa Solovita na condução das pesquisas. Expressamos agradecimentos ao produtor Toninho Pelicioni e ao Centro de Cana do IAC pelo empréstimo da infraestrutura experimental. 


\section{Referências}

CONAB - Companhia Nacional de Abastecimento. Acompanhamento da Safra Brasileira - Séries históricas, amendoim safra total, julho 2020. Disponível em: <https://www.conab.gov.br/info-agro/safras/graos>.

DEY, R.; PAL., K.K.; BHATT, D.M.; CHAUHAN, S.M. Growth promotion and yield enhancement of peanut (Arachis hypogaea $L$.) by application of plant growth-promoting rhizobacteria. Microbialogical Research, 159, p. 371394, 2004. https://doi.org/10.1016/j.micres.2004.08.004

DOLEY, K.; DUDHANE, M.; BORDE, M. Glomus fasciculatum in defense response to stop the rot of Arachis hypogaea L. Agrociência, v. 49, n. 6., p. 669-678, 2015.

ILLA, C.; PÉREZ, A.A.; TORASSA, M.; PÉREZ, M.A. Effect of biocontrol and promotion of peanut growth by inoculating Trichoderma harzianum and Bacillus subtilis under controlled conditions and field. Mexican Journal of

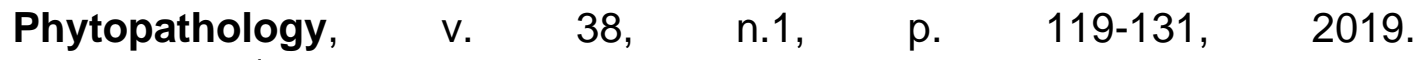
Doi10.18781/R.MEX.FIT.1910-6

PARNELL, J.J.; BERKA, R.; YOUNG, H.A.; STURINO, J.M.; KANG, Y.; BARNHART, D.M.; DILEO, M.W. From the Lab to the Farm: An Industrial Perspective of Plant Beneficial Microorganism. Frontiers in Plant Science, v. 7, Article 1110. http://dx.doi.org/10.3389/fpls.2016.01110

PODILE, A.R.; KISHORE,G.K. Biological Control of Peanut Diseases. In: Biological Control of Crop Diseases, Gnanamanickam, Samuel S. (ed.). CRC Press, 2019. P. 131-160.

REN, X.; ZHANG, Q.; ZHANG, W.; MAO, J.; LI, P. Control of aflatoxigenic molds by antagonistic microorganisms: Inhibitory behaviors, bioactive compounds, related mechanisms, and influencing factors. Toxins, v. 12, n. 24, p. 1-21, 2020. 10.3390/toxins12010024

SWANDI, F.; SULYANTI, E.; REFLIN, D. The potential of arbuscular mycorrhizal fungi (AM Fungi) as biocontrol agente against stem rot diseases caused by Sclerotium rolfsii in Peanut (Arachis hypogaea L.). Indonesian Journal of Crop Science, v. 2, n.2, p. 65-71, 2020.

VESSEY, J.K. Plant growth promoting rhizobacteria as biofertilizers. Plant and Soil, v. 255, p. 571-586, 2003. 